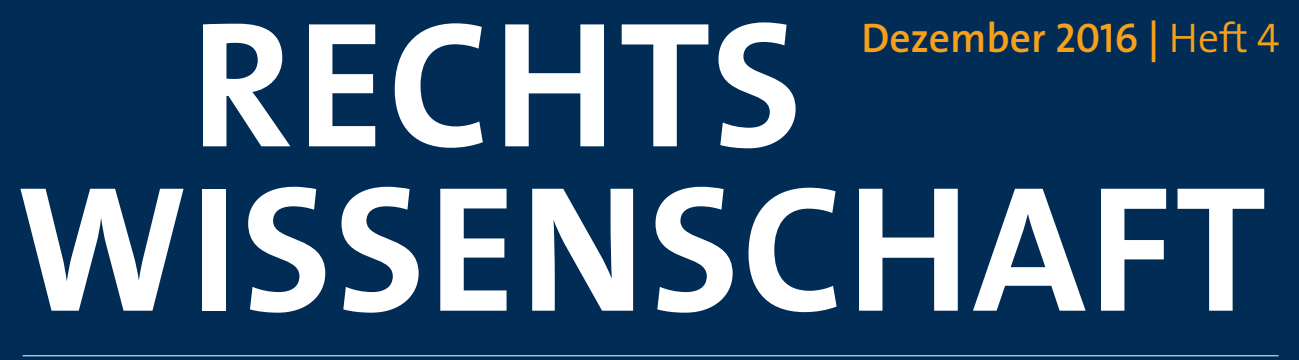

Zeitschrift für rechtswissenschaftliche Forschung

Herausgegeben von

Prof. Dr. Barbara Dauner-Lieb | Prof. Dr. Hans Christoph Grigoleit

Prof. Dr. Thomas Gutmann | Prof. Dr. Hans-Peter Haferkamp

Prof. Dr. Werner Heun | Prof. Dr. Tatjana Hörnle |

Prof. Dr. Urs Kindhäuser | Prof. Dr. Frank Neubacher

Prof. Dr. Anne Peters | Prof. Dr. Thomas Pfeiffer | Prof. Dr. Helmut Satzger

Prof. Dr. Helmuth Schulze-Fielitz | Prof. Dr. Ewald Wiederin

Prof. Dr. Joachim Wieland

Aus dem Inhalt

Rekonstruktion eines Staatsgeheimnisses

Jan-Hendrik Dietrich

Der deutsche Sozialstaat als Sozialversicherungsstaat und seine Auswirkungen auf das Präventionsrecht

Andrea Kießling

Versammlungsfreiheit und Privateigentum

Robert Frau 


\section{Zeitschrift für rechtswissenschaftliche Forschung}

Herausgegeben von Prof. Dr. Barbara Dauner-Lieb, Köln | Prof. Dr. Hans Christoph Grigoleit, München (LMU) | Prof. Dr. Thomas Gutmann, Münster | Prof. Dr. Hans-Peter Haferkamp, Köln | Prof. Dr. Dr. h.c. Werner Heun, Göttingen | Prof. Dr. Tatjana Hörnle, Berlin (HU) | Prof. Dr. Dr. h.c. Urs Kindhäuser, Bonn | Prof. Dr. Frank Neubacher M.A., Köln | Prof. Dr. Anne Peters LL.M., Heidelberg/ Basel | Prof. Dr. Thomas Pfeiffer, Heidelberg | Prof. Dr. Helmut Satzger, München (LMU) | Prof. Dr. Helmuth Schulze-Fielitz, Würzburg | Prof. Dr. Ewald Wiederin, Wien | Prof. Dr. Joachim Wieland, Speyer

Schriftleitung: Prof. Dr. Johannes Rux,Tübingen/Baden-Baden

\section{Inhaltsverzeichnis}

Johannes Rux

Danksagung und Begrüßung ....

\section{Abhandlungen}

Jan-Hendrik Dietrich

Rekonstruktion eines Staatsgeheimnisses

Andrea Kießling

Der deutsche Sozialstaat als Sozialversicherungsstaat und seine Auswirkungen

auf das Präventionsrecht

Robert Frau

Versammlungsfreiheit und Privateigentum

\section{Tagungen und ihre Folgen}

Andreas Holenstein/Rahel Aina Nedi

Arbeitsrecht und Arbeitswelt im europäischen Wandel

Till Zimmermann

Das 5. Symposium junger Strafrechtlerinnen und Strafrechtler am 29./30.4.2016 in Nürnberg 
Rezensionsaufsätze

Olaf Deinert

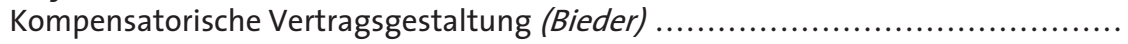

Carsten Herresthal

Gestaltungsfreiheit im Recht der Publikumsgesellschaft (Häusermann) .............

Jahresregister

Inhaltsverzeichnis nach Beitragsarten

Autorenverzeichnis

\section{Schriftleitung:}

Prof. Dr. Johannes Rux (V.i.S.d.P.) | Nomos Verlagsgesellschaft mbH \& Co. KG | Waldseestrasse 3-5 | D 76530 BadenBaden | Tel.: + 497221/210425 | Fax: + 497221/210427 | Mail: rux@nomos.de

Erscheinungsweise: 4 Ausgaben pro Jahr

Bezugspreise 2016: Jahresabonnement incl. Onlinezugang Privatbezieher 179,- $€$, Institutionen 289 ,- $€$, Einzelheft $44,-€$. Alle Preise verstehen sich incl. MWSt, zzgl. Vertriebskostenanteil.

Bestellmöglichkeit: Bestellungen beim örtlichen Buchhandel oder direkt bei der Nomos Verlagsgesellschaft BadenBaden

Kündigungsfrist: jeweils drei Monate vor Kalenderjahresende

Bankverbindung generell: Zahlungen jeweils im Voraus an Nomos Verlagsgesellschaft, Postbank Karlsruhe: DE07 660100750073636751 (IBAN), PBNKDEFF (BIC) oder Sparkasse Baden-Baden Gaggenau: DE05 66250030 0005002266 (IBAN), SOLADES1BAD (BIC)

Druck und Verlag: Nomos Verlagsgesellschaft mbH \& Co. KG | Waldseestrasse 3-5 | D-76530 Baden-Baden | Telefon (07221) 2104-o | Fax (07221) 2104-27 | E-Mail: nomos@nomos.de

Anzeigen: Sales friendly Verlagsdienstleistungen | Pfaffenweg 15 | 53227 Bonn | Telefon (0228) 978980 | Fax (0228) 9789820 | E-Mail: roos@sales-friendly.de

Urheber- und Verlagsrechte: Die Zeitschrift sowie alle in ihr enthaltenen einzelnen Beiträge und Abbildungen sind urheberrechtlich geschützt. Jede Verwertung, die nicht ausdrücklich vom Urheberrechtsgesetz zugelassen ist, bedarf der vorherigen Zustimmung des Verlags. Mit der Annahme zur Veröffentlichung überträgt der Autor dem Verlag das ausschließliche Verlagsrecht für die Zeit bis zum Ablauf des Urheberrechts. Eingeschlossen sind insbesondere auch das Recht zur Herstellung elektronischer Versionen und zur Einspeicherung in Datenbanken sowie das Recht zu deren Vervielfältigung und Verbreitung online oder offline ohne zusätzliche Vergütung. Nach Ablauf eines Jahres kann der Autor anderen Verlagen eine einfache Abdruckgenehmigung erteilen; das Recht an der elektronischen Version verbleibt beim Verlag. Namentlich gekennzeichnete Beiträge geben nicht in jedem Fall die Meinung der Herausgeber/Redaktion oder des Verlages wieder. Unverlangt eingesendete Manuskripte - für die keine Haftung übernommen wird - gelten als Veröffentlichungsvorschlag zu den Bedingungen des Verlages. Die Redaktion behält sich eine längere Prüfungsfrist vor. Eine Haftung bei Beschädigung oder Verlust wird nicht übernommen. Bei unverlangt zugesandten Rezensionsstücken keine Garantie für Besprechung oder Rückgabe. Es werden nur unveröffentlichte Originalarbeiten angenommen. Die Verfasser erklären sich mit einer nicht sinnentstellenden redaktionellen Bearbeitung einverstanden.

Der Nomos Verlag beachtet die Regeln des Börsenvereins des Deutschen Buchhandels e.V. zur Verwendung von Buchrezensionen.

ISSN $1868-8098$ 